\title{
Office diagnosis of pertussis
}

$\mathrm{O}^{2}$ UTBREAKS OF PERTUSSIS CONTINUE TO OCCUR IN CANADA despite adequate immunization rates in most communities. Cases occur in both unimmunized and immunized children, although the severity of illness appears to be less in the fully immunized child. Physicians providing care to children need to consider the possibility of pertussis in the child with a persistent cough as the diagnosis is clinical and laboratory confirmation may be difficult.

Initially, pertussis mimics the common cold. After the first week, however, the cough persists and becomes paroxysmal and may be terminated with vomiting or an inspiratory whoop. Paroxysmal cough or a 'non-improving' cough for two weeks should raise the suspicion of pertussis, particularly if there has been contact with a case of pertussis. Pertussis should also be considered in adults with recently acquired cough of two or more weeks duration.

Culture of the causative bacterium Bordetella pertussis from the nasopharynx is the only definitive laboratory confirmation, but is often difficult due to the fastidious nature of the organism. Peripheral blood lymphocytosis increases the suspicion of pertussis but is frequently absent. A direct fluorescent antibody test on respiratory secretions is available but has fallen into increasing disfavour because of the high rate of falsepositive and false-negative results with currently available reagents. ${ }^{1}$ Serological tests are receiving increasing attention but interpretation is often difficult because of the confounding effect of prior pertussis immunization. ${ }^{2}$ Presently, there is no commercially available, reliable serological test for the diagnosis of pertussis and these tests must still be considered research tools used by specialized laboratories.

Where does this leave the primary care physician attempting to confirm a clinical suspicion of pertussis? Culture of nasopharyngeal secretions remains the "gold

All material presented in Pediatric Infectious Disease Notes has been reviewed and approved by the chairperson, Canadian Pediatric Society Board and representative members of the Canadian Pediatric Society Committee on Infectious Diseases and Immunization standard' for laboratory diagnosis, and protocols for obtaining, transporting and processing specimens need to be arranged between physicians and microbiology laboratories.

$B$ pertussis is difficult to culture and physicians must be sure that the laboratory has a proven capacity either to isolate the organism or transport it to a reference facility. Cultures are not helpful if the paroxysmal cough has been present longer than three weeks or if erythromycin has been given for three days or more. ${ }^{2}$ Specimens can by obtained by nasopharyngeal swab using a calcium alginate swab (cotton swabs inhibit the growth of $B$ pertussis), or by nasopharyngeal aspirate using a fine (5 French) flexible plastic catheter connected to a $10 \mathrm{~mL}$ syringe. ${ }^{3}$ The latter technique ${ }^{4}$ has the advantage of decreasing the likelihood of drying of the specimen which greatly reduces recovery of the organism. If the specimen is taken at the same facility where the culture will be performed, the catheter can be transported to the laboratory for immediate culture. If the specimen is taken in the office, the catheter should be rinsed into a vial with $1 \mathrm{~mL}$ of sterile phosphate buffered saline with $1 \%$ casaminoacids prior to transport. This buffer can be supplied in vials by the laboratory and stored refrigerated for up to two months. Transport should be at refrigerated temperature if the specimen will take more than $4 \mathrm{~h}$ to reach the laboratory. Other transport media are available for $B$ pertussis, particularly if swabs are used rather than aspirates. These media require rapid transport to the laboratory and careful attention to transport temperature. $^{5}$

Several new tests being evaluated for the laboratory diagnosis of pertussis include detection of antibody in nasopharyngeal secretions, detection of pertussis toxin activity in secretions using a cytotoxicity assay, and detection of the $B$ pertussis genome in secretions by polymerase chain reaction. Whether these tests will be able to replace nasopharyngeal culture will depend on their sensitivity and specificity. Until new tests are better characterized and standardized, physicians will have to rely on their clinical acumen, and nasopharyngeal culture for the diagnosis of pertussis. 


\section{REFERENCES}

1. Halperin SA. Bortolussi R. Wort AS. Evaluation of culture, immunofluoresence, and serology for the diagnosis of pertussis. J Clin Microbiol 1989:27:752-75.

2. Onorato IM. Wassilak GF. Laboratory diagnosis of pertussis: The state of the art. Pediatr Infect Dis 1987;6;145-51.

3. Hoppe SE. Methods for isolation of Bordetella pertussis from patients with whooping cough. Eur J Clin Microbiol Infect Dis 1988:7:616-20.

4. Halperin SA, Kasina A, Swift M. Prolonged survival of
Bordetella pertussis in a simple buffer after nasopharyngeal secretion aspirate. Can J Microbiol. (In press)

5. Morrill WE, Barbaree JM, Fields BS, Sanden GN, Martin WT. Effects of transport temperature and medium on recovery of Bordetella pertussis from nasopharyngeal swabs. J Clin Microbiol 1988;26:1814-7.

Scott Halperin, MD Halifax, Nova Scotia 


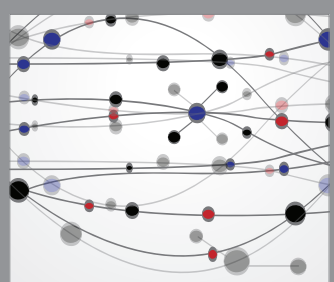

The Scientific World Journal
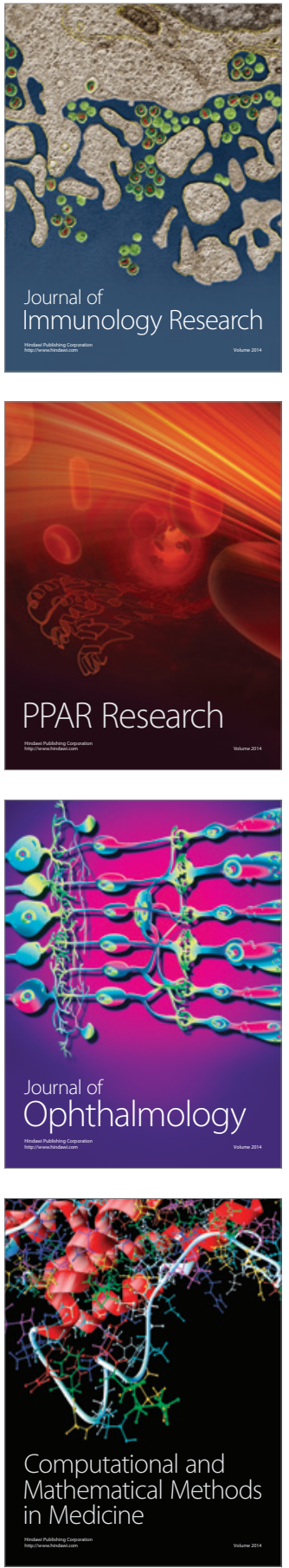

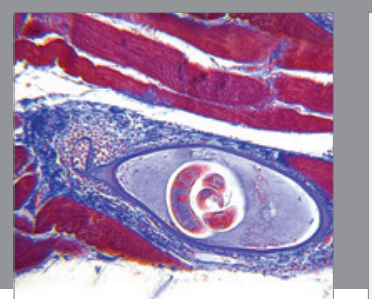

Gastroenterology Research and Practice

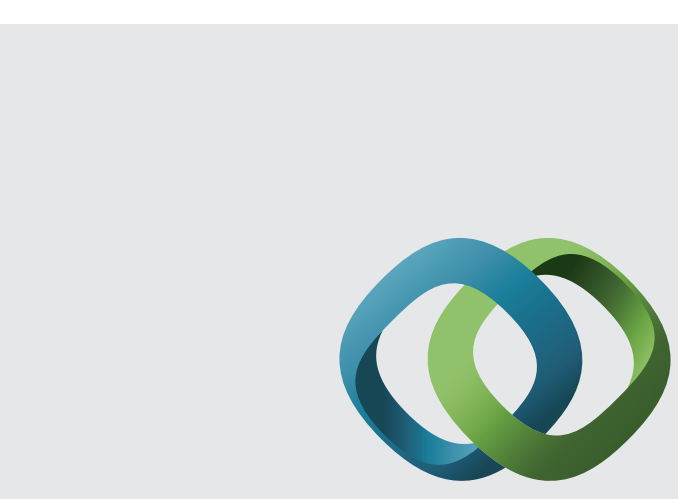

\section{Hindawi}

Submit your manuscripts at

http://www.hindawi.com
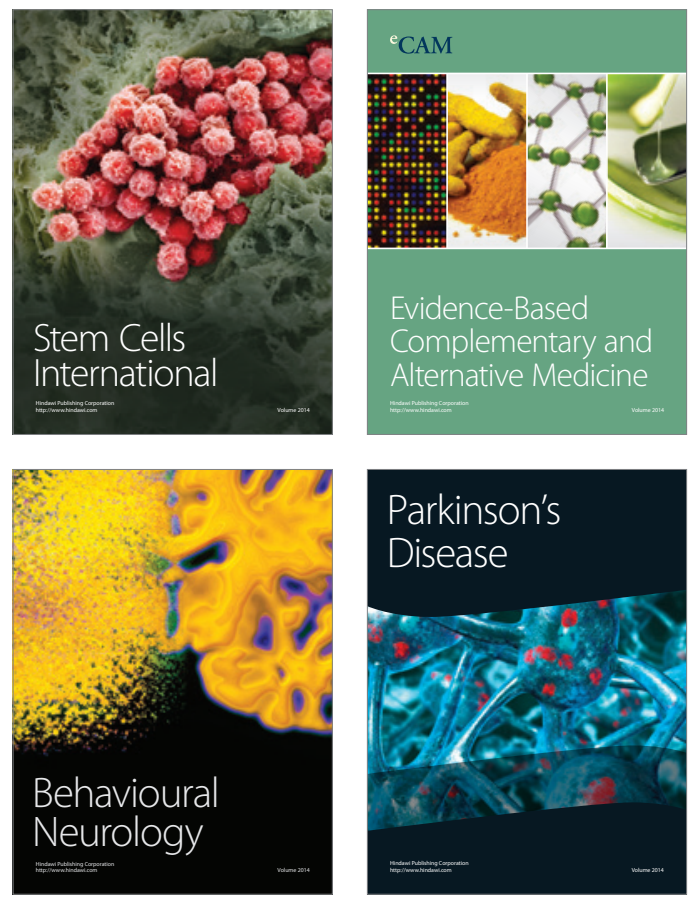
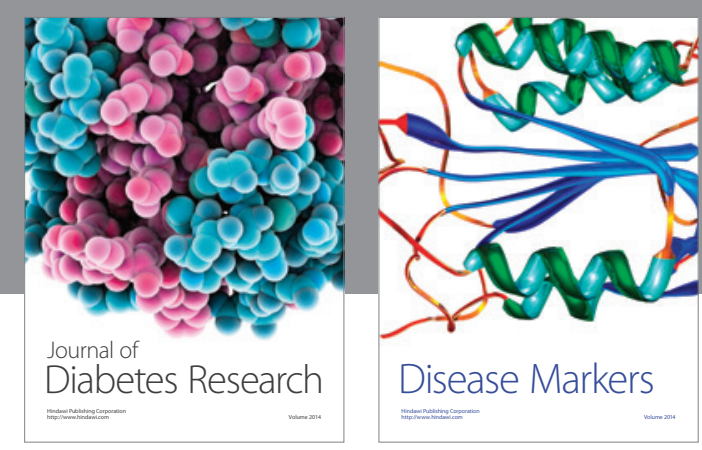

Disease Markers
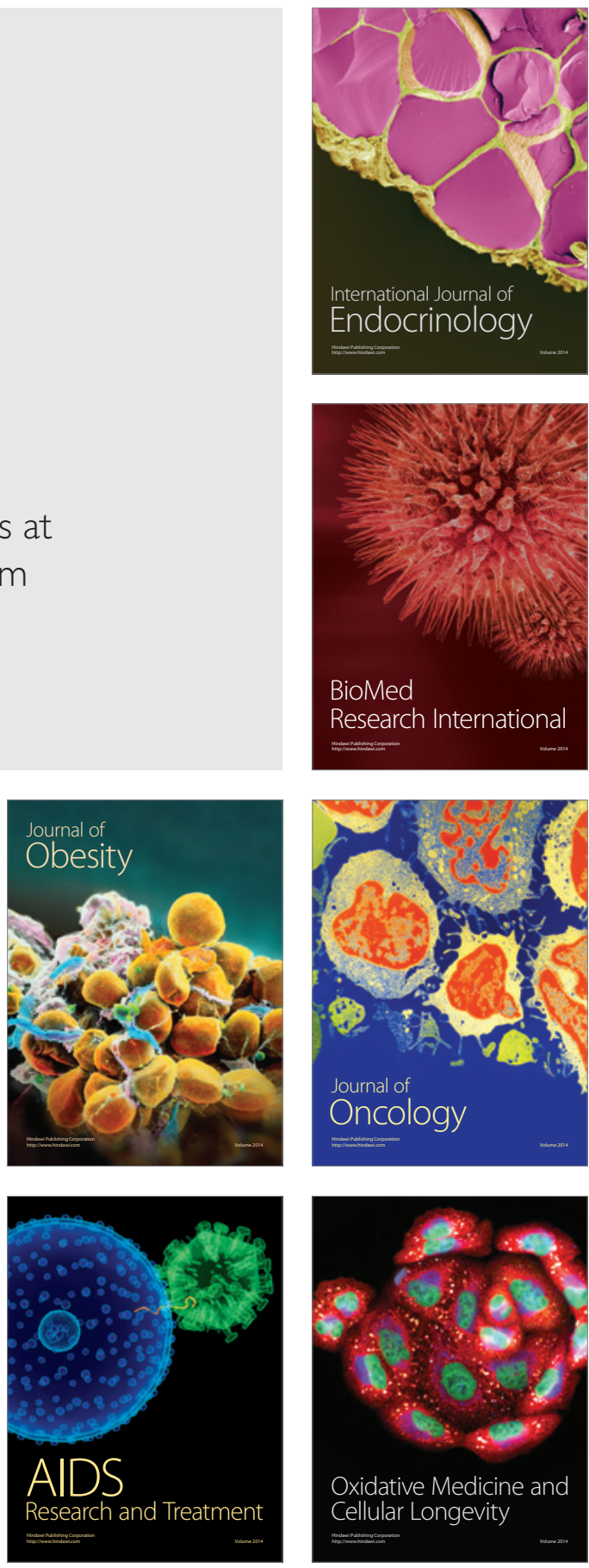